\title{
Disproportionate Pulmonary Hypertension in a Patient with Early-onset Pulmonary Emphysema Treated with Specific Drugs for Pulmonary Arterial Hypertension
}

\author{
Masatoshi Shimizu ${ }^{1}$, Junichi Imanishi ${ }^{1}$, Takatsugu Takano ${ }^{1}$ and Yoichi Miwa ${ }^{2}$
}

\begin{abstract}
Severe pulmonary hypertension in chronic obstructive pulmonary disease (COPD) is referred to as 'disproportionate' because the elevated pulmonary artery pressure does not match the degree of air flow limitation. We report a 41-year-old man presenting with early-onset pulmonary emphysema and pulmonary hypertension with a mean pressure of $74 \mathrm{mmHg}$. Continuous intravenous epoprostenol led to marked hemodynamic improvement, and epoprostenol was successfully replaced with bosentan. The patient has been followed for 3 years without exacerbation. This is the first report demonstrating the long-term efficacy of specific drugs for pulmonary arterial hypertension in disproportionate pulmonary hypertension in COPD.
\end{abstract}

Key words: early-onset pulmonary emphysema, pulmonary hypertension, epoprostenol, bosentan, chronic obstructive pulmonary disease

(Intern Med 50: 2341-2346, 2011)

(DOI: 10.2169/internalmedicine.50.5995)

\section{Introduction}

Definition of early-onset pulmonary emphysema includes disease onset before the age of 50 years irrespective of smoking history. If the disease is limited to Hugh-Jones classification of dyspnea higher than class II and forced expiratory volume in one second $\left(\mathrm{FEV}_{1}\right)<50 \%$ of predicted value, there is an estimated prevalence of less than 100 patients in Japan (1). Pulmonary hypertension occurs occasionally in patients with chronic obstructive pulmonary disease (COPD), and it is associated with a poor prognosis $(2,3)$. Pulmonary hypertension is relatively mild to moderate in most of the cases, but it could be very severe and 'disproportionate' to the degree of air flow limitation $(3,4)$. Therapeutic strategies for severe pulmonary hypertension include smoking cessation, pulmonary rehabilitation, and long-term oxygen supplementation, whereas the indications and effectiveness of specific drugs for pulmonary arterial hypertension remain controversial (2). We present a case of earlyonset pulmonary emphysema complicated by severe pulmo- nary hypertension. In the present case, continuous intravenous epoprostenol led to marked hemodynamic improvement, and epoprostenol was successfully replaced with bosentan in combination with beraprost without hemodynamic compromise.

\section{Case Report}

A 41-year-old man was admitted to the hospital because of dyspnea and general fatigue at rest in December 2007. He had experienced recurrent pneumothorax since the age of 25 . He had been diagnosed with pulmonary emphysema based on findings of a CT scan at the age of 32 when he was admitted to the hospital because of pneumothorax. In addition, echocardiography had revealed pulmonary hypertension with an estimated peak pressure gradient of 65 $\mathrm{mmHg}$. Until that time, he had smoked 20 cigarettes daily for 12 years, but he quit smoking ever since. He had been followed at another clinic with administration of inhaled anticholinergics, theophylline, and short-acting beraprost (120 $\mu \mathrm{g} / \mathrm{day})$. However, he developed exertional dyspnea at the

${ }^{1}$ Department of Cardiology, National Hospital Organization Kobe Medical Center, Japan and ${ }^{2}$ Department of Internal Medicine, National Hospital Organization Kobe Medical Center, Japan

Received for publication June 14, 2011; Accepted for publication July 19, 2011

Correspondence to Dr. Masatoshi Shimizu, cheetahms@hera.eonet.ne.jp 

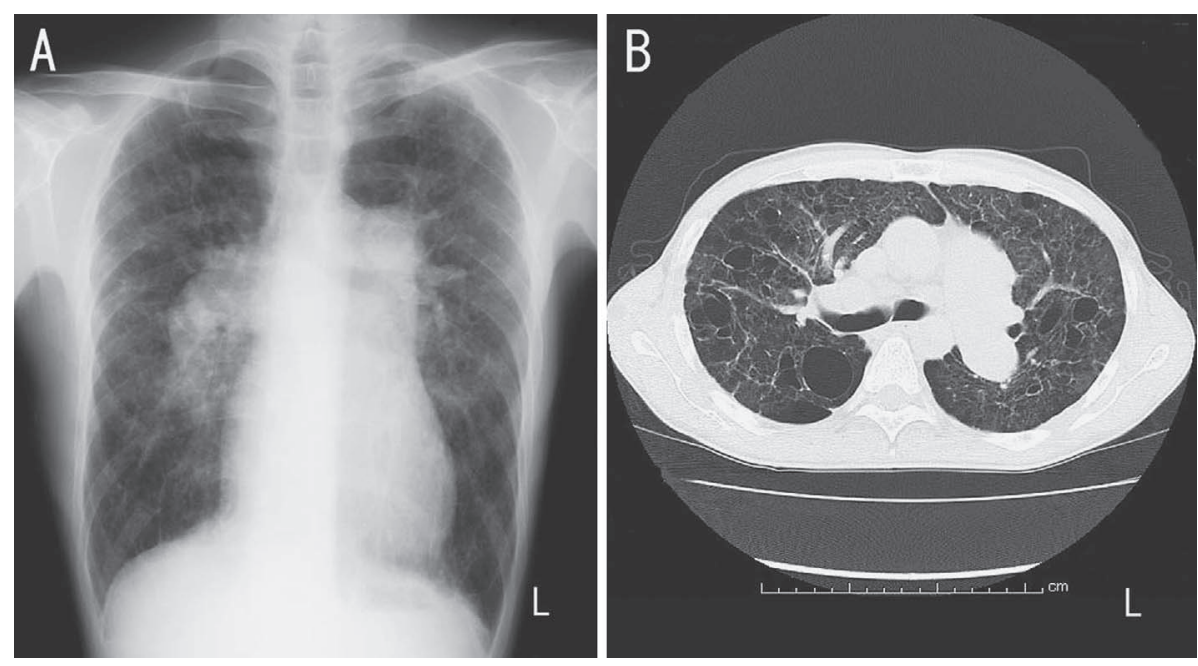

Figure 1. Anteroposterior chest radiograph (panel A) and computed tomography of the chest (panel B). The chest radiograph at admission shows enlarged hilar pulmonary arteries indicating pulmonary arterial hypertension. Computed tomography of the lungs demonstrates panlobular emphysema, which is an exceptional finding considering the patient's age.

age of 39 , which progressively worsened to such a degree that he became dyspneic even at rest (Hugh-Jones grade IV). He was referred to our hospital for further management. He had no family history of pulmonary emphysema in first-degree relatives. He had not been engaged in any occupation related to irritant particles or gases, and denied using illegal drugs. On admission, his heart rate was 120 beats/ $\mathrm{min}$, and blood pressure was 120/86 mmHg. His pulse oximetry saturation was $88 \%$ on room air. The body mass index was $17.1 \mathrm{~kg} / \mathrm{m}^{2}(171 \mathrm{~cm}, 50 \mathrm{~kg})$. Auscultation revealed a grade III systolic murmur with an accentuated second pulmonary sound. Prolonged expiration without any wheezes or rhonchi was noted. Laboratory findings were unremarkable, except for a uric acid of $8.1 \mathrm{mg} / \mathrm{L}$ and brain natriuretic peptide (BNP) of $361 \mathrm{pg} / \mathrm{dL}$. Both KL-6 and $\alpha 1$-antitrypsin were within normal range. Arterial-blood-gas analysis showed a pH of 7.45, a $\mathrm{PCO}_{2}$ of 35 Torr, and a $\mathrm{PaO}_{2}$ of 46 Torr during breathing of room air. Pulmonary function tests showed severe airflow obstruction and impaired diffusion capacity: forced vital capacity (FVC) $2.56 \mathrm{~L}$ (64.9\% of predicted value), $\mathrm{FEV}_{1} 1.15 \mathrm{~L}$ ( $32.6 \%$ of predicted value), $\mathrm{FEV}_{1} / \mathrm{FVC}$ ratio $46.7 \%$, residual volume $2.81 \mathrm{~L}$ (171.3\% of predicted value) and carbon monoxide diffusion capacity (DLCO) $37.0 \%$ of predicted value. Given the patient's age, these findings fulfilled the criteria of early-onset pulmonary emphysema. An electrocardiogram revealed pulmonary $\mathrm{P}$, right axis deviation, $\mathrm{qR}$ pattern in $\mathrm{V}_{1}$, and clockwise rotation in precordial leads. A chest radiograph demonstrated enlarged pulmonary arteries and inflated lung fields (Fig. 1A). Computed tomography of the chest showed panlobular emphysema (Fig. 1B). A ventilation-perfusion lung scan showed no features of mismatched defect. A 6-minute walk test had to be discontinued in 2 minutes with a distance of less than $100 \mathrm{~m}$. Echocardiography showed marked right ventricular dilatation with hypertrophy and flattened inter- ventricular septum (Fig. 2A-C). No congenital abnormalities of the heart were found. Doppler echocardiography revealed an estimated right ventricular systolic pressure of 98.4 mmHg (mean: $60.1 \mathrm{mmHg}$ ) (Fig. 2D). The patient underwent right heart catheterization, which demonstrated mean right atrial pressure $8 \mathrm{mmHg}$, mean pulmonary arterial pressure $74 \mathrm{mmHg}$, pulmonary wedge pressure $10 \mathrm{mmHg}$, cardiac output $3.92 \mathrm{~L} / \mathrm{min}$, and pulmonary vascular resistance 1,265 dyne $\cdot \mathrm{sec} \cdot \mathrm{cm}^{-5}$. The patient received $2.5 \mathrm{~L} / \mathrm{min}$ of nasal oxygen, oral anticoagulation therapy, dobutamine, and human atrial natriuretic peptide. He was categorized as WHO functional class IV, and we considered that his symptoms were mainly due to severe pulmonary arterial hypertension. Then, intravenous epoprostenol infusion was initiated, the dose of which was gradually increased from $1 \mathrm{ng} / \mathrm{kg} / \mathrm{min}$ to $19 \mathrm{ng} / \mathrm{kg} / \mathrm{min}$. The treatment with intravenous epoprostenol for 6 months resulted in marked improvement in exertional dyspnea and pulmonary hemodynamics (Table 1). The 6minute walk distance increased to $420 \mathrm{~m}$, and follow-up right heart catheterization showed mean pulmonary arterial pressure $49 \mathrm{mmHg}$, cardiac output $4.72 \mathrm{~L} / \mathrm{min}$, and pulmonary vascular resistance 891 dyne $\cdot \mathrm{sec} \cdot \mathrm{cm}^{-5}$. The BNP level was $18.9 \mathrm{pg} / \mathrm{dL}$. However, the patient developed psychiatric symptoms such as restlessness, a sense of palpitation, and a growing feeling of anxiety. He was prescribed antidepressants. Oral sildenafil was started at dosage of $40-60 \mathrm{mg}$ in addition to epoprostenol, whereas sildenafil had to be discontinued after 3 months because the psychiatric problems became rather worse. We started bosentan therapy and attempted to gradually withdraw intravenous epoprostenol. The initial dosage of $125 \mathrm{mg}$ bosentan was increased to 250 mg after 5 days. Fortunately, the patient was successfully weaned off intravenous epoprostenol without worsening of his symptoms. The psychiatric symptoms were almost relieved after withdrawal of epoprostenol, and the patient was 

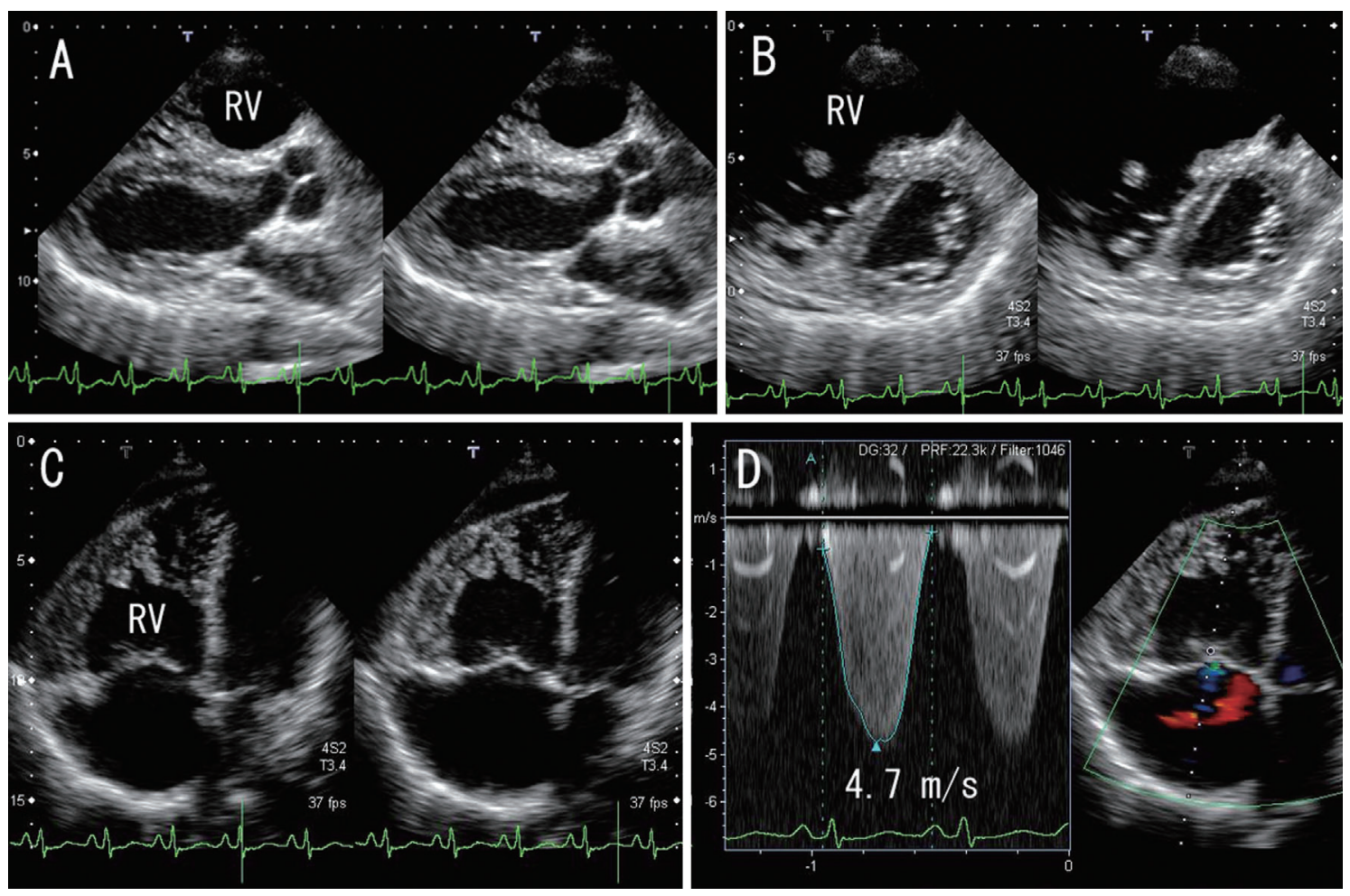

Figure 2. Echocardiographic examinations. Panels A, B and C show marked right ventricular dilatation (left: end-diastole, right: end-systole). Panel B demonstrates flat interventricular septum in the cross-sectional view, and panel $\mathrm{C}$ demonstrates hypertrophied right ventricular free wall in the apical four chamber view. Panel D shows that the peak velocity of tricuspid regurgitation is $4.7 \mathrm{~m} / \mathrm{s}$, suggesting an estimated right ventricular systolic pressure of $98.4 \mathrm{mmHg}$ (mean: $60.1 \mathrm{mmHg}$ ). RV: right ventricle

Table 1. The Clinical Course of the First 14 Months

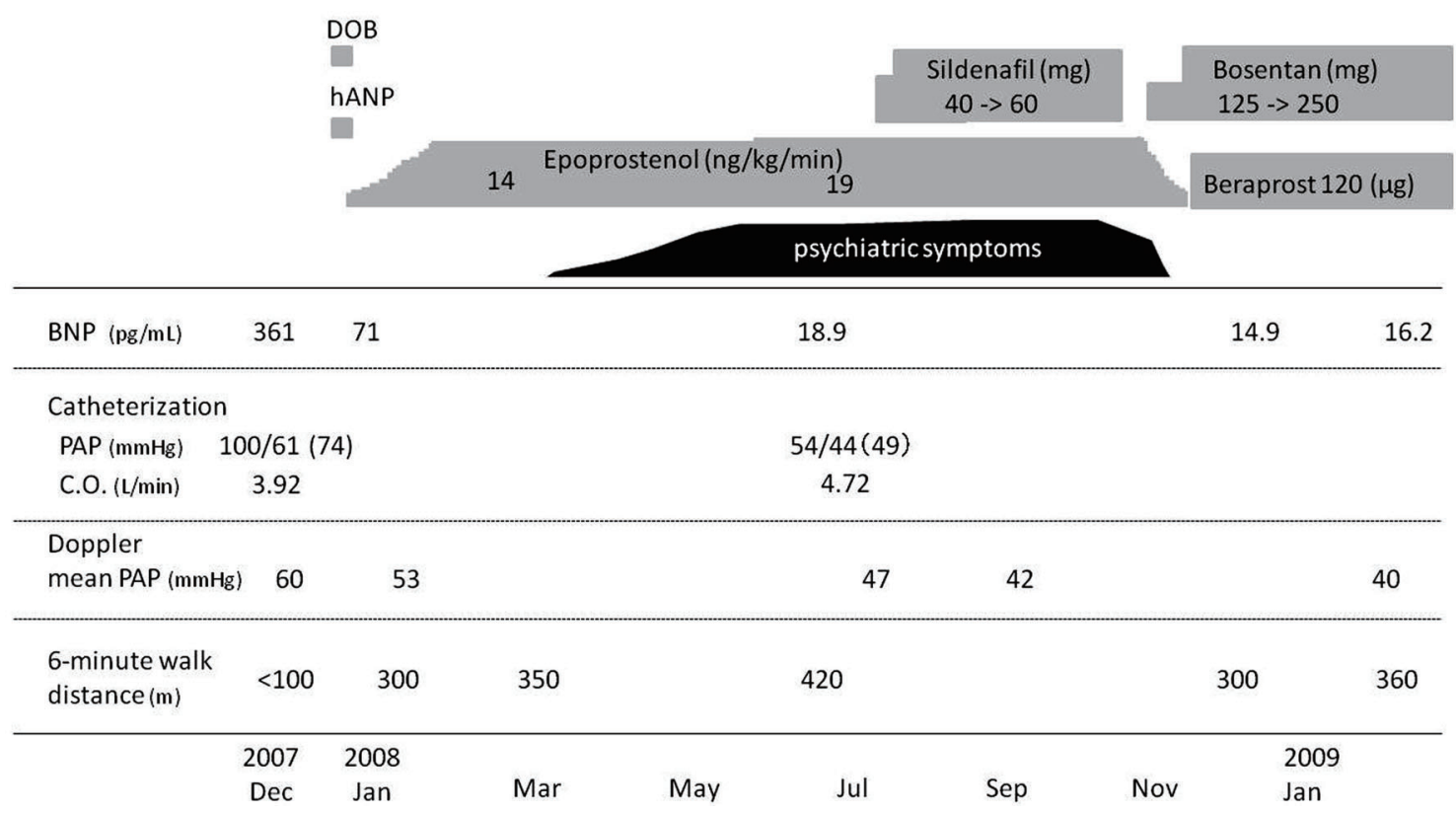

DOB: dobutamine, hANP: human atrial natriuretic peptide, BNP: brain natriuretic peptide, PAP: pulmonary arterial pressure, C.O.: cardiac output

supplemented with long-acting oral beraprost $(120 \mu \mathrm{g} / \mathrm{day})$. The 6-minute walk distance was $360 \mathrm{~m}$ after 2 months of bosentan and beraprost treatment. A follow-up echocar- diography showed that right ventricular dilatation and flattened interventricular septum became less prominent (Fig. 3). Mean pulmonary artery pressure was $40 \mathrm{mmHg}$. 

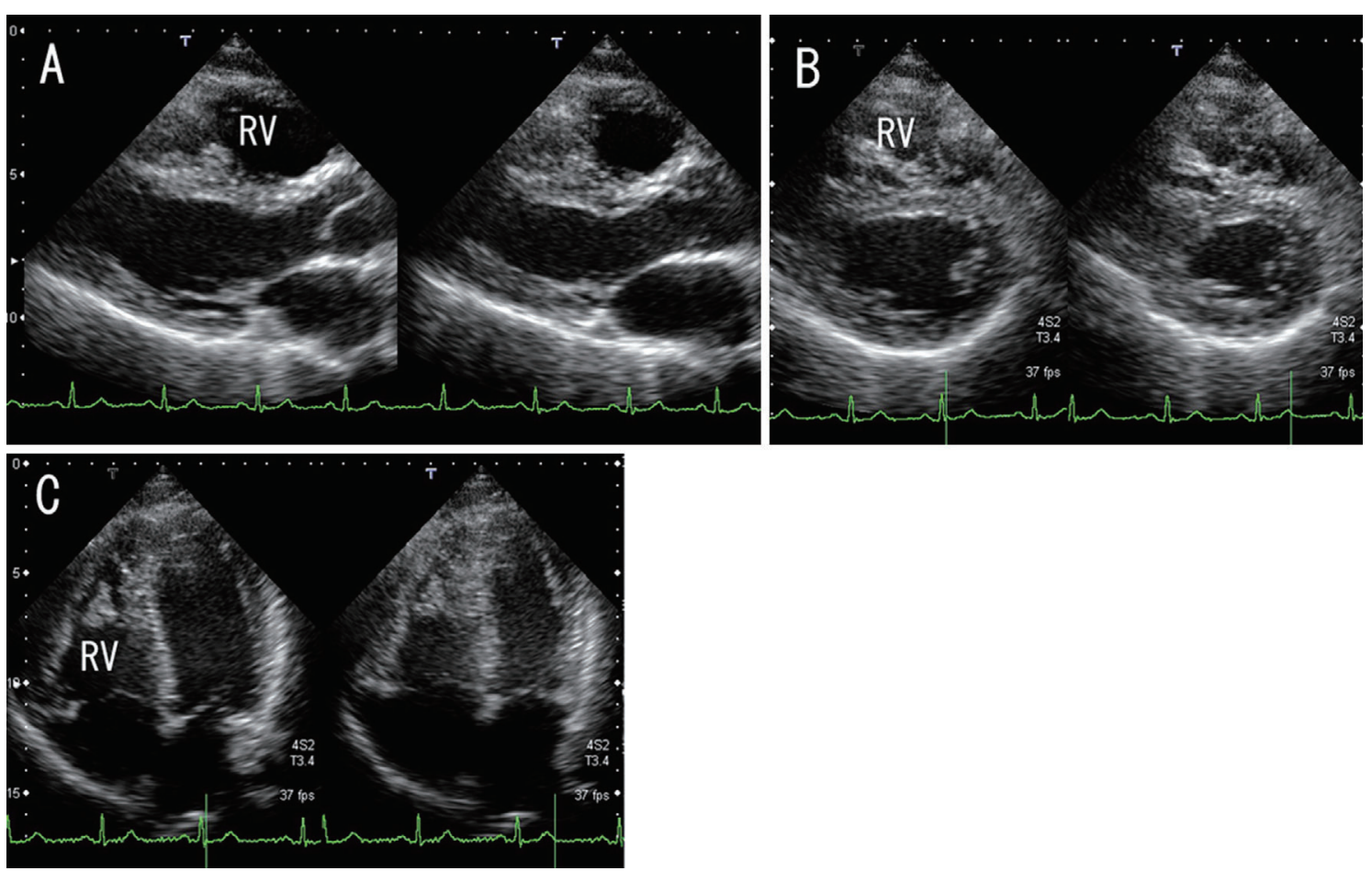

Figure 3. Follow-up echocardiograms show that right ventricular dilatation is reduced and the interventricular septal curvature is less flattened. A: Parasternal long-axis view. B: Parasternal shortaxis view. C: Apical four chamber view.

The patient returned to his previous job as a machine designer under bosentan, beraprost, and long-term oxygen therapy. He has been followed for 3 years without exacerbation of symptoms. His oxygen saturation at rest was maintained at $94 \%$, and Doppler-estimated mean pulmonary artery pressure remained at less than $50 \mathrm{mmHg}$.

\section{Discussion}

Severe pulmonary hypertension constitutes an important clinical problem in patients with COPD. The present patient suffered from severe pulmonary hypertension with a mean pressure of $76 \mathrm{mmHg}$, which was not explained by left sided heart failure, congenital heart disease, or pulmonary embolism. Although pulmonary hypertension occurs occasionally in patients with COPD, most of them have only mild to moderate pulmonary hypertension (2). Severe pulmonary hypertension with a mean pressure $>40 \mathrm{mmHg}$ occurs in only $0.8 \%$ to $3.7 \%$ of COPD patients (3-6). It is characterized by less impaired respiratory function, and the elevated pulmonary pressure does not correlate with the degree of air flow limitation $(3,4)$. Because the elevated pulmonary arterial pressures do not correlate with the severity of COPD, it is called disproportionate pulmonary hypertension in COPD. Chaouat et al reported that the patients with disproportionate pulmonary hypertension had less severe airflow limitation, decreased DLCO, more severe hypoxemia, and hypocapnia (3). As for the present patient, the markedly elevated pulmonary arterial pressure is disportionate to his $\mathrm{FEV}_{1}$ which is not in the most advanced stage of COPD. In addition, the present patient had a very low DLCO, and blood gas analysis demonstrated marked hypoxemia requiring oxygen therapy and hypocapnia. Therefore, the severe pulmonary hypertension in the present patient is compatible with disproportionate pulmonary hypertension in COPD.

The mechanism of severe pulmonary hypertension in COPD remains not fully elucidated. Alveolar hypoxia, inflammation and genetic predisposition have been postulated as underlying causes of extensive vascular remodeling which leads to elevated pulmonary arterial pressure. Alveolar hypoxia causes constriction of resistance pulmonary arteries, and a close relationship has been reported between the severity of arterial hypoxemia and pulmonary vascular resistance $(4,6)$. However, arterial $\mathrm{PO}_{2}$ was not an independent predictor of pulmonary artery pressure, suggesting the involvement of other factors (6). Recently, genetic factors have been identified that predispose to pulmonary hypertension. For example, single nucleotide polymorphisms from surfactant protein B are linked to pulmonary artery pressure in patients with severe COPD (7). The present patient had a smoking history of 240 pack years, when he was diagnosed with emphysema and moderate pulmonary hypertension at the age of 32. The pulmonary arterial pressure continued to rise even after the cessation of smoking. We believe that some genetic predisposition can be involved in the severe pulmonary hypertension in the present case.

While specific drugs for pulmonary arterial hypertension, including endothelin receptor antagonists, phosphodiesterase5 inhibitors, and prostanoids have been tried in treating COPD-related pulmonary hypertension, their effectiveness 
has not been established yet. Pulmonary hypertension associated with COPD has some histological similarities to idiopathic pulmonary arterial hypertension. Pathologic studies of lung specimens from patients with COPD have shown extensive vascular remodeling with prominent intimal thickening, medial hypertrophy, and muscularization of small arterioles $(8,9)$. These findings have prompted clinicians to treat pulmonary hypertension in COPD with specific drugs for pulmonary arterial hypertension. Stolz et al reported that the endothelin receptor antagonist bosentan not only failed to improve exercise capacity but also deteriorated hypoxemia and functional status in severe COPD patients without severe pulmonary hypertension at rest (10). In a study of the phosphodiesterase-5 inhibitor sildenafil, Blanco et al concluded that sildenafil improved pulmonary hemodynamics at rest and during exercise in patients with COPD-associated pulmonary hypertension, while these effects were accompanied by impaired arterial oxygenation due to the inhibition of hypoxic vasoconstriction (11). Since these studies included patients with only mild to moderate pulmonary hypertension or exercise-induced pulmonary hypertension, it is important to note that the results may not apply to severe pulmonary hypertension in COPD. Some reports have shown improvement in functional status and hemodynamic parameters after bosentan therapy in COPD patients with severe pulmonary hypertension (12-14). Oxygenation and cardiac output were well maintained after bosentan therapy. These results are supported by the fact that hypoxic pulmonary hypertension is characterized by an overproduction of endothelin-1, which causes vasoconstriction and vessel wall thickening (15). However, the long-term efficacy of bosentan for severe pulmonary hypertension in COPD has not been shown.

We initially administered continuous intravenous epoprostenol with supplemental oxygen to the present patient, as epoprostenol is the first-line therapy for patients with pulmonary arterial hypertension in NYHA/WHO functional class IV (16). Treatment with epoprostenol for six months resulted in marked improvement of BNP level, 6-minute walk distance, pulmonary arterial pressure, and pulmonary arterial resistance. However, the patient became intolerable to intravenous epoprostenol due to psychiatric symptoms. Intravenous epoprostenol treatment is complex and requires dedicated management by the patient, such as sterile preparation of the medication and aseptic management of the indwelling central venous catheter. Because epoprostenol has a very short half-life (2.7 minutes), abrupt discontinuation of the drug may lead to potentially life-threatening complications. These difficulties of intravenous epoprostenol treatment could cause substantial emotional stress for the patient. The addition of oral sildenafil therapy to epoprostenol did not lead to further clinical improvement but rather exacerbated psychiatric problems. We started bosentan therapy in place of sildenafil. Bosentan allowed successful withdrawal from epoprostenol without hemodynamic impairment (17). Long-acting oral beraprost was used as combination therapy.
Although the 6-minute walk distance decreased by $60 \mathrm{~m}$, the psychiatric problems were almost relieved after epoprostenol discontinuation. Our experience with the present case suggests that specific drugs for pulmonary arterial hypertension such as epoprostenol and bosentan could be effective to severe pulmonary hypertension in COPD. Although some detrimental effects on gas exchange are to be expected with pulmonary vasodilation, his oxygen saturation at rest was maintained at $94 \%$ under long-term oxygen therapy. COPD patients with severe pulmonary hypertension have a very poor prognosis $(2,3)$. The survival of the present patient for more than 3 years would not have been achieved if epoprostenol and bosentan had not been applied. This is the first report demonstrating the long-term efficacy of specific drugs for pulmonary arterial hypertension in severe pulmonary hypertension in COPD.

We would like to stress that specific drugs for pulmonary arterial hypertension may not be applicable to severe COPD patients with less severe pulmonary hypertension, because pulmonary vasodilators also have the potential to worsen gas exchange and oxygenation $(10,11)$. These drugs should be used carefully in patients with severe pulmonary hypertension irrespective of the severity of COPD. Furthermore, as far as we have searched the literature, there are no reports demonstrating the effectiveness of sildenafil for severe pulmonary hypertension in COPD. It will be necessary to obtain further evidence before the use of specific drugs for pulmonary arterial hypertension can be recommended for severe pulmonary hypertension in COPD.

The authors state that they have no Conflict of Interest (COI).

\section{References}

1. Fujimoto K, Kubo K. The epidemiology of early onset COPD in Japan: a nationwide cross-sectional study of presenting features and prognostic factors. Research report of respiratory failure research group of the Japanese Ministry of Health, Labor, and Welfare 2007: 25-30, 2008 (in Japanese).

2. Minai OA, Chaouat A, Adnot S. Pulmonary hypertension in COPD: epidemiology, significance, and management: pulmonary vascular disease: the global perspective. Chest 137: 39S-51S, 2010.

3. Chaouat A, Bugnet AS, Kadaoui N, et al. Severe pulmonary hypertension and chronic obstructive pulmonary disease. Am J Respir Crit Care Med 172: 189-194, 2005.

4. Thabut G, Dauriat G, Stern JB, et al. Pulmonary hemodynamics in advanced COPD candidates for lung volume reduction surgery or lung transplantation. Chest 127: 1531-1536, 2005.

5. Stevens D, Sharma K, Szidon P, Rich S, McLaughlin V, Kesten S. Severe pulmonary hypertension associated with COPD. Ann Transplant 5: 8-12, 2000.

6. Scharf SM, Iqbal M, Keller C, Criner G, Lee S, Fessler HE; National Emphysema Treatment Trial (NETT) Group. Hemodynamic characterization of patients with severe emphysema. Am J Respir Crit Care Med 166: 314-322, 2002.

7. Castaldi PJ, Hersh CP, Reilly JJ, Silverman EK. Genetic associations with hypoxemia and pulmonary arterial pressure in COPD. Chest 135: 737-744, 2009.

8. Magee F, Wright JL, Wiggs BR, Paré PD, Hogg JC. Pulmonary 
vascular structure and function in chronic obstructive pulmonary disease. Thorax 43: 183-189, 1988.

9. Santos S, Peinado VI, Ramírez J, et al. Characterization of pulmonary vascular remodelling in smokers and patients with mild COPD. Eur Respir J 19: 632-638, 2002.

10. Stolz D, Rasch H, Linka A, et al. A randomised, controlled trial of bosentan in severe COPD. Eur Respir 32: 619-628, 2008.

11. Blanco I, Gimeno E, Munoz PA, et al. Hemodynamic and gas exchange effects of sildenafil in patients with chronic obstructive pulmonary disease and pulmonary hypertension. Am J Respir Crit Care Med 181: 270-278, 2010.

12. Cottin V, Khouatra C, Lazor R, Canu P, Cordier JF. To the Editors. Eur Respir J 33: 450-452, 2009.

13. Figueroa VR, Pérez AH, Lecha EA, Cancelo IR, Ramos DV. Bosentan in pulmonary hypertension associated with hypoxaemic lung diseases. Curr Med Res Opin 23: S49-S53, 2007.

14. Valerio G, Bracciale P, Grazia D'Agostino A. Effect of bosentan upon pulmonary hypertension in chronic obstructive pulmonary disease. Ther Adv Respir Dis 3: 15-21, 2009.

15. Faller M, Kessler R, Sapin R, et al. Regulation of endothelin-1 at rest and during a short steady-state exercise in 21 COPD patients. Pulm Pharmacol Ther 11: 151-157, 1998.

16. Galiè N, Hoeper MM, Humbert M, et al. Task Force for Diagnosis and Treatment of Pulmonary Hypertension of European Society of Cardiology (ESC); European Respiratory Society (ERS); International Society of Heart and Lung Transplantation (ISHLT). Guidelines for the diagnosis and treatment of pulmonary hypertension. Eur Heart J 30: 2493-2537, 2009.

17. Johnson RF, Loyd JE, Mullican AL, Fink CA, Robbins IM. Longterm follow-up after conversion from intravenous epoprostenol to oral therapy with bosentan or sildenafil in 13 patients with pulmonary arterial hypertension. J Heart Lung Transplant 26: 363-369, 2007.

(C) 2011 The Japanese Society of Internal Medicine http://www.naika.or.jp/imindex.html 